\title{
Dual-Energy CT Angiography Improves Accuracy of Spot Sign for Predicting Hematoma Expansion in Intracerebral Hemorrhage
}

\author{
Michaël T.J. Peeters, ${ }^{a}$ Kim J.D. de Kort, ${ }^{a}$ Rik Houben, ${ }^{a}$ Wouter J.P. Henneman, ${ }^{\text {b }}$ Robert J. van Oostenbrugge, ${ }^{\mathrm{a}}$ \\ Julie Staals, ${ }^{\text {a Alida A. Postma }}{ }^{\mathrm{b}}$ \\ ${ }^{a}$ Department of Neurology and Cardiovascular Research Institute Maastricht, Maastricht University Medical Center, Maastricht, Netherlands \\ ${ }^{b}$ Department of Radiology and Nuclear Medicine, MHeNS School for Mental Health and Neuroscience, Maastricht University Medical Center, \\ Maastricht, Netherlands
}

Background and Purpose Spot sign (SS) on computed tomography angiography (CTA) is associated with hematoma expansion (HE) and poor outcome after intracerebral hemorrhage (ICH). However, its predictive performance varies across studies, possibly because differentiating hyperdense hemorrhage from contrast media is difficult. We investigated whether dual-energy-CTA (DE-CTA), which can separate hemorrhage from iodinated contrast, improves the diagnostic accuracy of SS for predicting HE.

Methods Primary ICH patients undergoing DE-CTA (both arterial as well as delayed venous phase) and follow-up computed tomography were prospectively included between 2014 and 2019. SS was assessed on both arterial and delayed phase images of the different DE-CTA datasets, i.e., conventional-like mixed images, iodine images, and fusion images. Diagnostic accuracy of SS for prediction of $\mathrm{HE}$ was determined on all datasets. The association between SS and $\mathrm{HE}$, and between SS and poor outcome (modified Rankin Scale at 3 months $\geq 3$ ) was assessed with multivariable logistic regression, using the dataset with highest diagnostic accuracy.

Results Of 139 included patients, 47 showed HE (33.8\%). Sensitivity of SS for HE was 32\% (accuracy 0.72 ) on conventional-like mixed arterial images which increased to $76 \%$ (accuracy 0.80 ) on delayed fusion images. Presence of SS on delayed fusion images was independently associated with $\mathrm{HE}$ (odds ratio [OR], 17.5; 95\% confidence interval [Cl], 6.14 to 49.82) and poor outcome (OR, 3.84; $95 \% \mathrm{Cl}, 1.16$ to 12.73$)$.

Conclusions Presence of SS on DE-CTA, in particular on delayed phase fusion images, demonstrates higher diagnostic performance in predicting HE compared to conventional-like mixed imaging, and it is associated with poor outcome.

Keywords Computed tomography angiography; Cerebral hemorrhage; Intracranial hemorrhages; Spot sign; Prognosis
Correspondence: Michaël T.J. Peeters Department of Neurology and Cardiovascular Research Institute Maastricht, Maastricht University Medical Center, P. Debyelaan 25, $6225 \mathrm{HX}$ Maastricht, Netherlands Tel: $+31-433876500$ Fax: $+31-433877615$ E-mail: maikel.peeters@mumc.nl https://orcid.org/0000-0002-63752264

Received: August 12, 2020 Revised: October 17, 2020 Accepted: October 22, 2020

\section{Introduction}

Intracerebral hemorrhage (ICH) carries a high mortality rate and burden of morbidity. Most predictors of outcome in $\mathrm{ICH}^{1}$, such as age, Glasgow Coma Scale (GCS) score, initial hematoma volume and hematoma location, are unmodifiable., In contrast, 
significant hematoma expansion (HE), which occurs in up to a third of patients with $\mathrm{ICH}$, is a strong and potentially modifiable predictor of poor functional outcome and mortality. ${ }^{4.5}$

The spot sign (SS), defined as one or more spots of enhancement within an acute hematoma due to active contrast extravasation on computed tomography angiography (CTA), has been shown to predict HE as well as clinical outcome. ${ }^{6}$ However, varying CTA acquisition techniques, such as arterial phase or delayed (venous) phase imaging, result in a wide range of diagnostic accuracy. Two meta-analysis showed a rather low pooled sensitivity of $57 \%$ and $62 \%$ of the SS for predicting HE., ${ }^{2,7}$

Assessment of SS on CTA might be challenging as it is difficult to differentiate hyperdense hemorrhage from contrast agent. Dual-energy CTA (DE-CTA) is a technique that uses high- and low-peak voltage acquisitions simultaneously. ${ }^{8,9}$ Because of the difference in energy-dependent attenuation curves, DE-CTA can distinguish between hemorrhage and iodinated contrast media. ${ }^{10}$ We hypothesized that the application of DE-CTA, including both arterial and delayed phase imaging, improves the diagnostic accuracy of the SS for predicting $\mathrm{HE}$ in patients with primary ICH when compared to conventional-like arterial and delayed phase imaging.

\section{Methods}

\section{Patient selection and image acquisition}

We prospectively included patients (aged 18 years or older) with imaging confirmed primary $\mathrm{ICH}$ admitted to the Maastricht University Medical Center (MUMC), the Netherlands, between January 1st, 2014 and December 31st, 2019. The study was approved by the medical ethical committee of the MUMC. Written informed consent for collection and analysis of clinical data was obtained if possible, but the requirement for consent was waived in case a patient died before consent or when the patient was incapacitated by the stroke and no legal representative was available.

Patients with traumatic ICH, secondary ICH including vascular malformations, hemorrhagic transformation of ischemic stroke and neoplasms as well as non-parenchymal hemorrhage (e.g., epidural, subdural, subarachnoid, and primary intraventricular hemorrhage [IVH]) were not included. We excluded patients who did not undergo DE-CTA and/or follow-up computed tomography (CT) within 48 hours. Patients were excluded if the delay between baseline brain CT and DE-CTA was $>3$ hours. Patients who underwent surgical evacuation of the hematoma before follow-up CT were also excluded from analysis. Patients lacking follow-up CT, but otherwise fulfilling inclusion criteria, were included in the secondary analysis of functional outcome.

\section{Patient characteristics}

Clinical data including age, sex, time of last seen well (LSW), GCS at admission, anticoagulant use, systolic blood pressure at admission, glucose at admission, and cardiovascular risk factors were collected. Functional outcome was assessed at 3 months on the modified Rankin Scale (mRS) and was obtained either through standardized interviews or outpatient clinical contact. Poor outcome was defined as $m R S \geq 3$.

\section{Image acquisition and post-processing}

As part of standard care in patients with $\mathrm{ICH}$, non-contrast brain CT was followed by DE-CTA at arterial phase as well as an 80-second delayed phase. Non-contrast and DE-CTA imaging was obtained using a second-generation dual-source CT system (Somatom Definition Flash or Somatom Definition Force, Siemens Healthcare, Forcheim, Germany). Non-contrast CT (both primary CT as well as follow-up CT) were acquired from skull base to the cranial vertex with the following parameters: 120 peak kilovoltage $(\mathrm{kVp}), 340$ reference milliampereseconds (ref. mAs), slice thickness/spacing $1.0 \mathrm{~mm}$, computed tomography dose index (CTDI) $34 \mathrm{mGy}$. Patients then received $90 \mathrm{~mL}$ intravenous contrast media (Ultravist $300 \mathrm{mg} / \mathrm{mL}$, Bayer Healthcare, Leverkusen, Germany) with a flow of $5 \mathrm{~mL} / \mathrm{sec}$. The hereafter performed dedicated dual-source protocol comprised of arterial phase imaging (80/140 kVp, 135/68 ref. mAs, CTDI $7.86 \mathrm{mGy})$ and 80 -second delayed phase imaging (80/140 kVp, $550 / 275$ ref. mAs, CTDI $25.34 \mathrm{mGy}$ ) with rotation time set at 0.28 seconds and $2 \times 64 \times 0.6 \mathrm{~mm}$ collamination.

Conventional-like mixed images, which simulate single-energy acquisitions at a typical kVp of 120, were generated by blending data from the high-and low energy component with a weighted average of $0.6 .^{11}$ These conventional-like mixed images were generated for both the arterial phase (mixed arterial) and delayed phase (mixed delayed). Post-processing took place using SyngoVia (Siemens Healthcare) to generate iodine maps (arterial iodine and delayed iodine) using three-material decomposition. ${ }^{8,12,13}$ Fusion images were created by registration and overlaying of the iodine with the mixed images (arterial fusion and delayed fusion). Examples of the different imaging datasets are presented in Figure 1. Non-enhanced follow-up brain CT was performed within 48 hours after the primary brain CT.

\section{Imaging analysis}

All DE-CTA datasets were analyzed by an experienced neuroradiologist (A.A.P.) with $>10$ years of experience, who was blinded to clinical data and HE status. Per protocol consecutively the non-enhanced brain CT, mixed arterial phase, mixed delayed phase, arterial phase iodine, arterial phase fusion, delayed 

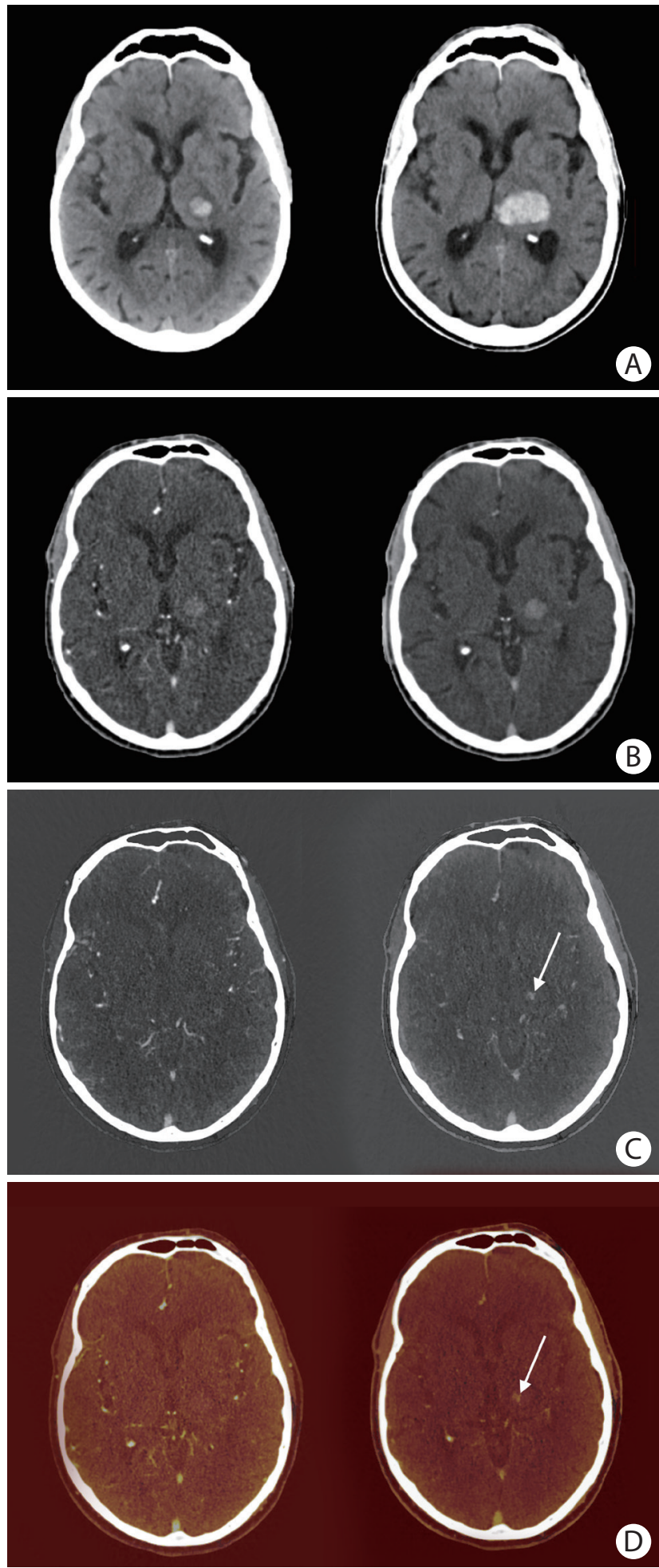

Figure 1. An 81-year old man with primary intracerebral hemorrhage $(\mathrm{ICH})$ in the left thalamus. (A) Non-contrast baseline computed tomography (CT) (left) and non-contrast follow-up CT after 24 hours (right). (B) Mixed arterial image (left) and mixed delayed image (right). (C) Arterial iodine image (left) and delayed iodine image (right) with spot sign (SS) (arrow). (D) Arterial fusion image (left) and delayed fusion image (right) with SS (arrow). Arterial images did not show a SS. A SS can clearly be seen on delayed iodine ( $C$, right panel) and fusion images ( $D$, right panel), but could not be distinguished from hyperintense hemorrhage signal on conventional-like mixed delayed image ( $B$, right panel). In this case, significant hematoma expansion took place (A). phase iodine, and delayed phase fusion images were assessed (Figure 1). SS was defined as $\geq 1$ foci of contrast within the hematoma and discontinuous from surrounding blood vessels. Hematoma location was classified as supratentorial or infratentorial. The interobserver agreement for identifying the SS was calculated with the Cohen's kappa coefficient (k). After assessment of 30 randomly picked cases by two neuroradiologists (A.A.P., W.J.P.H.) the interobserver agreement was lowest, but still substantial for mixed arterial and mixed delayed phase imaging (both $\mathrm{k}$ of 0.62), which is comparable to a previous study. ${ }^{14}$ The interobserver agreement improved on the DE datasets, reaching near perfect on arterial phase iodine and delayed phase fusion datasets ( $\mathrm{k}$ of 0.77 and 0.84 , respectively).

Baseline and follow-up ICH volumes were measured using a semi-automated volume segmentation technique in SyngoVia (Siemens Healthcare). When semi-automated volume determination was not possible (e.g., due to technical issues, $n=33$ ), $\mathrm{ICH}$ volumes were calculated using the validated $\mathrm{ABC} / 2$ method. ${ }^{15} \mathrm{IVH}$ was excluded from volume measurements. Significant $\mathrm{HE}$ was defined as a volume increase of $>6 \mathrm{~mL}$ or an increase of $>33 \%$ from baseline ICH volume. ${ }^{5,6}$

\section{Statistical analysis}

Statistical analysis was performed using SPSS for Windows version 25.0 (IBM Corp., Amonk, NY, USA). To test for differences between groups we used chi-square test for categorical data and Mann-Whitney test for non-normally distributed data.

Sensitivity, specificity, positive predictive value, negative predictive value (NPV), and accuracy of the SS for HE were calculated for the different DE-CTA datasets separately as well as for presence of the SS on any of these DE-CTA datasets. Receiver operating characteristic (ROC) curves were computed and area under the curve (AUC) was calculated. A value of $P \leq 0.05$ was considered significant.

Univariable logistic regression analyses was used to test for associations between HE (dependent variable) and demographic, clinical and radiological variables, process measures and SS on DE-CTA dataset that showed highest accuracy. Age, sex, SS, and those variables associated with $\mathrm{HE}$ in univariable regression analysis at a $P<0.10$ level, were subsequently analyzed in multivariable logistic regression analysis.

Both univariable and multivariable logistic regression analyses were performed to test for associations between poor outcome (dependent variable) and age, sex, SS on DE-CTA dataset that showed highest accuracy and other well-known predictors of outcome (anticoagulant use, systolic blood pressure at admission, glucose at admission, GCS, baseline ICH volume, presence of IVH on first CT-scan, and infratentorial ICH location). 


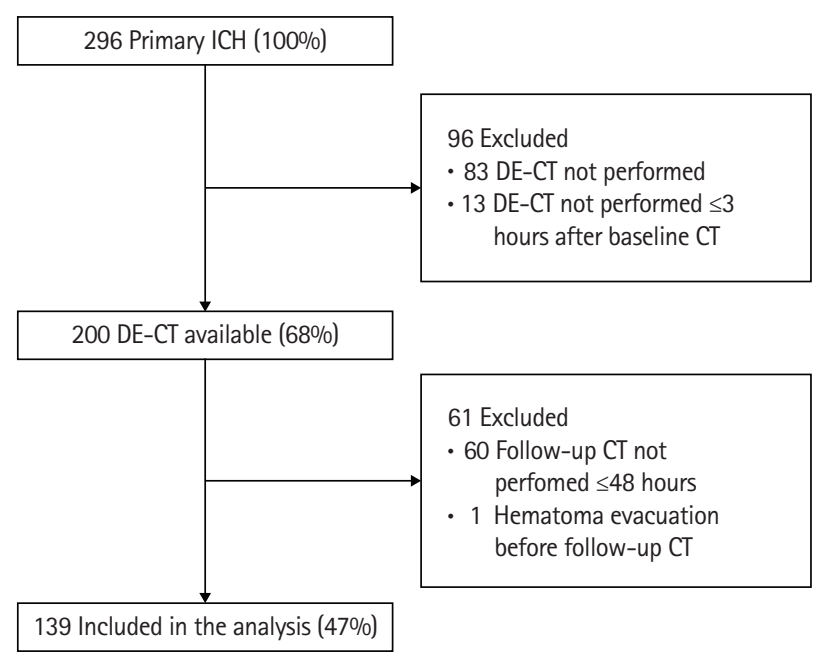

Figure 2. Flow-chart of selection study cohort. ICH, intracerebral hemorrhage; $\mathrm{DE}-\mathrm{CT}$, dual-energy computed tomography; $\mathrm{CT}$, computed tomography.

\section{Results}

\section{Study population}

A total of 296 primary ICH patients presented to our emergency department between 2014 and 2019. Ninety-six patients were excluded, either because no DE-CTA was performed $(n=83)$ or DE-CTA was performed $\geq 3$ hours after baseline CT $(n=13)$ (Figure 2). These excluded patients $(n=96)$ more often were female, were older, had a higher serum glucose on admission, showed a larger baseline hematoma volume with more frequent intraventricular expansion, presented with lower GCS, and more frequently had poor outcome, when compared to patients who were included (Supplementary Table 1). Most frequently reported reason for not performing DE-CTA were very poor prognosis on presentation or a diminished renal function.

We further excluded 60 patients because they did not receive follow-up CT $\leq 48$ hours (due to poor clinical status or demise), and one patient who underwent hematoma evacuation before follow-up CT was performed (Figure 1). We includ-

Table 1. Baseline characteristics

\begin{tabular}{|c|c|c|c|c|c|}
\hline Characteristic & $\begin{array}{l}\text { Data complete } \\
\text { (\% of total) }\end{array}$ & $\begin{array}{l}\text { Total group } \\
(\mathrm{n}=139)\end{array}$ & $\begin{array}{l}\text { Hematoma expansion } \\
\qquad(n=47)\end{array}$ & $\begin{array}{l}\text { No hematoma expansion } \\
\qquad(n=92)\end{array}$ & $P$ \\
\hline \multicolumn{6}{|l|}{ Clinical } \\
\hline Age (yr) & $139(100)$ & $76.0(65.0-82.0)$ & $76.0(62.0-82.0)$ & $75.5(67.0-82.0)$ & 0.957 \\
\hline Male sex & $139(100)$ & $88(63.3)$ & $32(68.1)$ & $56(60.9)$ & 0.404 \\
\hline History of hypertension & 139 (100) & $91(65.5)$ & $30(63.8)$ & $61(66.3)$ & 0.772 \\
\hline Hypercholesterolemia & 138 (99.3) & $54(38.8)$ & $22(46.8)$ & $32(34.8)$ & 0.184 \\
\hline Anticoagulant treatment & $139(100)$ & $33(23.7)$ & $17(36.2)$ & $16(17.4)$ & 0.014 \\
\hline Glucose (mmol/L) & $137(98.6)$ & $6.7(5.9-8.5)$ & $7.0(5.8-8.9)$ & $6.7(5.9-8.2)$ & 0.611 \\
\hline Systolic blood pressure $(\mathrm{mm} \mathrm{Hg})$ & $139(100)$ & $170(155-194)$ & $170(155-189)$ & 173 (155-195) & 0.779 \\
\hline GCS & $139(100)$ & $15(13-15)$ & $15(13-15)$ & $15(13-15)$ & 0.946 \\
\hline mRS after 3 months & $126(90.6)$ & $4(2-6)$ & $6(4-6)$ & $4(2-6)$ & 0.021 \\
\hline \multicolumn{6}{|l|}{ Imaging } \\
\hline Infratentorial ICH location & $139(100)$ & $9(6.5)$ & $4(8.5)$ & $5(5.4)$ & 0.486 \\
\hline Baseline ICH volume (mL) & $139(100)$ & $12.0(5.5-28.3)$ & $18.0(6.9-36.5)$ & $10.9(4.1-19.8)$ & 0.055 \\
\hline Presence of IVH on first CT-scan & $139(100)$ & 47 (33.8) & $19(40.4)$ & $28(30.4)$ & 0.239 \\
\hline SS on $\geq 1$ of the DE-CT acquisitions & $126(90.6)$ & $57(45.2)$ & 37 (78.7) & $20(21.7)$ & $<0.001$ \\
\hline SS on mixed arterial & $139(100)$ & $21(15.1)$ & 15 (31.9) & $6(6.5)$ & $<0.001$ \\
\hline SS on mixed delayed & $138(99.3)$ & $46(33.3)$ & $29(61.7)$ & $17(18.5)$ & $<0.001$ \\
\hline SS on arterial iodine & $134(96.4)$ & $21(15.7)$ & $16(34.0)$ & $5(5.4)$ & $<0.001$ \\
\hline SS on arterial fusion & $131(94.2)$ & $23(17.6)$ & 17 (36.2) & $6(6.5)$ & $<0.001$ \\
\hline SS on delayed iodine & $127(91.4)$ & $48(37.8)$ & $33(70.2)$ & $15(16.3)$ & $<0.001$ \\
\hline SS on delayed fusion & $122(87.8)$ & $46(37.7)$ & $32(68.1)$ & $14(15.2)$ & $<0.001$ \\
\hline \multicolumn{6}{|l|}{ Process measures } \\
\hline Time from LSW to imaging (DE-CT) (hr) & $136(97.8)$ & $4.0(2.0-14.0)$ & $4.0(1.0-8.5)$ & $4.0(2.0-17.0)$ & 0.022 \\
\hline Time from DE-CT to follow-up CT (hr) & $139(100)$ & $21.0(16.0-24.0)$ & $21.0(13.0-27.0)$ & $21.0(17.0-24.0)$ & 0.917 \\
\hline
\end{tabular}

Values are presented as median (interquartile range) or number (\%).

GCS, Glasgow Coma Scale; mRS, modified Rankin Scale; ICH, intracerebral hemorrhage; IVH, intraventricular hemorrhage; CT, computed tomography; SS, spot sign; DE-CT, dual-energy computed tomography; LSW, last seen well. 
Table 2. Performance of the spot sign for predicting hematoma expansion

\begin{tabular}{|c|c|c|c|c|c|c|}
\hline Variable & Sensitivity (\%) & Specificity (\%) & PPV (\%) & NPV (\%) & Accuracy & AUC $(95 \% \mathrm{Cl})$ \\
\hline SS on mixed arterial $(n=139)$ & 31.91 & 93.48 & 71.43 & 72.88 & 0.73 & $0.64(0.53-0.75)$ \\
\hline SS on mixed delayed $(n=138)$ & 61.70 & 81.32 & 63.04 & 80.43 & 0.75 & $0.72(0.61-0.82)$ \\
\hline SS on arterial iodine $(n=134)$ & 35.56 & 94.38 & 76.19 & 74.34 & 0.75 & $0.66(0.55-0.77)$ \\
\hline SS on arterial fusion $(n=131)$ & 39.53 & 93.18 & 73.91 & 75.93 & 0.76 & $0.66(0.56-0.77)$ \\
\hline SS on delayed iodine $(n=127)$ & 73.33 & 81.71 & 68.75 & 84.81 & 0.79 & $0.79(0.69-0.88)$ \\
\hline SS on delayed fusion $(n=122)$ & 76.19 & 82.50 & 69.57 & 86.84 & 0.80 & $0.79(0.70-0.88)$ \\
\hline SS on $\geq 1$ of the DE-CT acquisition(s) $(n=126)$ & 82.22 & 75.31 & 64.91 & 88.41 & 0.78 & $0.79(0.70-0.88)$ \\
\hline
\end{tabular}

PPV, positive predictive value; NPV, negative predictive value; $A U C$, area under the curve; $\mathrm{Cl}$, confidence interval; SS, spot sign; DE-CT, dual-energy computed tomography.

Table 3. Univariable and multivariable logistic regression analysis for variables associated with hematoma expansion

\begin{tabular}{|c|c|c|c|c|}
\hline \multirow{2}{*}{$\begin{array}{l}\text { Outcome: hematoma expansion } \\
\qquad(n=139)\end{array}$} & \multicolumn{2}{|c|}{ Univariable analysis } & \multicolumn{2}{|c|}{ Multivariable analysis } \\
\hline & $\mathrm{OR}$ & $95 \% \mathrm{Cl}$ & $\mathrm{OR}^{*}$ & $95 \% \mathrm{Cl}$ \\
\hline Age & 1.00 & $0.97-1.03$ & 0.98 & $0.94-1.02$ \\
\hline Male sex & 1.37 & $0.65-2.88$ & 2.01 & $0.68-5.94$ \\
\hline Hypertension & 0.90 & $0.43-1.87$ & & \\
\hline Hypercholesterolemia $(n=138)$ & 1.62 & $0.79-3.32$ & & \\
\hline Anticoagulant treatment & 2.69 & $1.21-6.01$ & 2.44 & $0.69-8.60$ \\
\hline Glucose $(\mathrm{mmol} / \mathrm{L})(\mathrm{n}=137)$ & 1.05 & $0.90-1.22$ & & \\
\hline Systolic blood pressure $(\mathrm{mm} \mathrm{Hg})$ & 1.00 & $0.99-1.01$ & & \\
\hline GCS & 1.02 & $0.86-1.21$ & & \\
\hline Time from LSW to imaging (DE-CT) $(n=136)$ & 0.95 & $0.92-1.00$ & 0.97 & $0.93-1.01$ \\
\hline Baseline ICH volume & 1.01 & $1.00-1.03$ & 1.01 & $0.99-1.03$ \\
\hline SS delayed fusion $(n=122)$ & 15.08 & $6.04-37.66$ & 17.49 & $6.14-49.82$ \\
\hline
\end{tabular}

$\mathrm{OR}$, odds ratio; $\mathrm{Cl}$, confidence interval; GCS, Glasgow Coma Scale; LSW, last seen well; DE-CT, dual-energy computed tomography; ICH, intracerebral hemorrhage; SS, spot sign.

*Multivariable regression analysis including associated variables derived from univariable regression analysis having a $P<0.1$ ( $n=120$ cases in the multivariable analysis).

Table 4. Univariable and multivariable logistic regression analysis for poor outcome (mRS $\geq 3)^{*}$

\begin{tabular}{|c|c|c|c|c|}
\hline \multirow{2}{*}{ Outcome: poor outcome $(n=179)$} & \multicolumn{2}{|c|}{ Univariable analysis } & \multicolumn{2}{|c|}{ Multivariable analysis } \\
\hline & $\mathrm{OR}$ & $95 \% \mathrm{Cl}$ & $\mathrm{OR}^{+}$ & $95 \% \mathrm{Cl}$ \\
\hline Age & 1.02 & $0.99-1.05$ & 1.00 & $0.96-1.05$ \\
\hline Male sex & 0.49 & $0.24-1.02$ & 0.36 & $0.12-1.04$ \\
\hline Anticoagulant treatment & 2.57 & $1.01-6.57$ & 3.17 & $0.84-11.98$ \\
\hline Glucose (mmol/L) (n=173) & 1.31 & $1.06-1.61$ & 1.38 & $0.98-1.95$ \\
\hline Systolic blood pressure $(\mathrm{mm} \mathrm{Hg})(\mathrm{n}=178)$ & 1.00 & $0.99-1.01$ & 1.01 & $0.99-1.03$ \\
\hline GCS & 0.97 & $0.93-1.02$ & 0.47 & $0.27-0.84$ \\
\hline Baseline ICH volume & 1.04 & $1.02-1.07$ & 1.04 & $1.00-1.08$ \\
\hline Presence of IVH on first CT-scan & 4.44 & $1.85-10.65$ & 1.39 & $0.35-5.47$ \\
\hline Infratentorial ICH location & 2.80 & $0.61-12.73$ & 5.49 & $0.81-37.52$ \\
\hline SS delayed fusion ( $n=154)$ & 5.24 & $2.14-12.82$ & 3.84 & $1.16-12.73$ \\
\hline
\end{tabular}

mRS, modified Rankin Scale; OR, odds ratio; Cl, confidence interval; GCS, Glasgow Coma Scale; ICH, intracerebral hemorrhage; IVH, intraventricular hemorrhage; $\mathrm{CT}$, computed tomography; SS, spot sign.

*Sixty patients without control $\mathrm{CT} \leq 48$ hours and one patient with hematoma evacuation are re-included; ${ }^{+} n=149$ cases in the multivariable analysis. 
ed 139 patients for analyses. Baseline characteristics of included patients are presented in Table 1.

\section{Spot sign and hematoma expansion}

A total of 47 patients showed HE (33.8\%). Patients with HE more often used anticoagulants and had shorter window from LSW to DE-CTA. Furthermore, patients with HE had a poorer functional outcome at 3 months.

Presence of SS on the different DE-CTA datasets is presented in Table 1. Not all DE-CTA datasets could be constructed in all patients. SS was more often detected on delayed phase imaging compared to first pass imaging, in all datasets (mixed, iodine, fusion). HE was significantly more frequent in all SS positive patients compared to SS negative patients.

Table 2 presents performance of SS on different DE-CTA datasets for predicting HE. Sensitivity of SS for HE increased from $31.9 \%$ on mixed arterial phase imaging to $76.2 \%$ on delayed phase fusion imaging. Accuracy was highest for delayed phase fusion imaging $(0.80 ; 95 \%$ confidence interval [Cl], 0.70 to 0.88; $P<0.001)$.

Table 3 presents the associations between HE (dependent variable) and age, sex, cardiovascular risk factors, anticoagulant use, systolic blood pressure at admission, GCS, baseline ICH volume, time from LSW to DE-CTA imaging, and SS on delayed phase fusion imaging. In univariable regression analysis the SS on delayed phase fusion was associated with HE (odds ratio [OR], 15.08; 95\% $\mathrm{Cl}, 6.04$ to 37.66 ), as were anticoagulant use, shorter time from LSW to imaging (DE-CTA) and larger baseline ICH volume. In multivariable regression analysis, the SS on delayed phase fusion imaging was independently associated with $\mathrm{HE}(\mathrm{OR}, 17.5 ; 95 \% \mathrm{Cl}, 6.14$ to 49.82$)$.

\section{Spot sign and poor outcome}

To assess the association between SS and poor outcome, we reincluded patients fulfilling the inclusion criteria of this study but lacking follow-up CT $\leq 48$ hours after baseline CT ( $n=60)$ and one patient who underwent hematoma evacuation before follow-up CT. Clinical outcome after 3 months was available in 179 patients. A total of 133 patients (66.5\%) had poor outcome after 3 months ( $m R S \geq 3$ ). Both univariable and multivariable logistic regression analyses showed an independent association between poor outcome and presence of SS on delayed phase fusion imaging. These findings are summarized in Table 4.

\section{Discussion}

In this study we found that the application of DE-CTA, and more specifically the evaluation of delayed phase fusion imag- es, improves the diagnostic accuracy of the SS for HE when compared to conventional-like mixed arterial and mixed delayed phase imaging. We demonstrated a sensitivity of 32\% of the SS on mixed arterial phase, which increased to $76 \%$ for a SS on delayed phase fusion. In addition, we showed that the presence of SS on delayed phase fusion is independently associated with poor clinical outcome.

Studies on the application of DE-CT in the detection of SS in ICH are sparse. A retrospective small study in 36 patients with primary and traumatic ICHs, showed that DE-CT facilitates SS detection and is able to differentiate iodine contrast from hematoma when compared to a delayed phase enhanced CT (2 minutes after contrast injection). HE was not analyzed. ${ }^{9}$ Another study used a machine learning approach on DE-CT acquisitions in primary $\mathrm{ICH}$ and identified two quantitative markers of $\mathrm{HE}$, which were combined in a HE probability score. The HE probability score showed better accuracy for prediction of HE compared to the conventional SS as assessed on single-energy $\mathrm{CT}^{9,16} \mathrm{Al}-$ though this study used an automatic iodine quantification method, while we visually assessed SS, its rationale to use DE-CT for improved detection of SS in predicting HE is likewise.

The independent association between SS and HE has been shown in various other studies. Reported sensitivity of the SS for predicting HE ranges from 21\% to 93\%, with two recent meta-analyses showing a pooled sensitivity of $62 \%$ and $57 \%$ respectively. ${ }^{24,7,17-25}$ We found a low sensitivity of $32 \%$ of the SS on conventional-like mixed arterial phase imaging for predicting HE. While we set no limitation in the onset or LSW-toscan time in order to not exclude late expanders, many former studies only included patients with an onset-to-scan time $\leq 6$ hours. A former study found a $60 \%$ sensitivity of SS for HE if CTA took place within 2 hours of onset, declining to $30 \%$ in patients with onset-to-scan time $>8$ hours. The sensitivity of SS for HE therefore seems to be inversely related to onset-toscan time. ${ }^{26}$ Furthermore, varying definitions of HE as well as the difficulty of differentiating hyperdense hemorrhage from contrast media extravasation could contribute to the differences in sensitivity. In the most used definition of SS (an attenuation $\geq 120$ Hounsfield units), the differentiation of SS from surrounding ICH (having attenuation of 30 to 90 Hounsfield units) can often be reliably established on single-energy CTA. However, subtle contrast extravasation is more difficult to differentiate from surrounding $\mathrm{ICH}$ on single-energy CTA, but nevertheless is clinically important. A previous study found that a $>10 \%$ increase in Hounsfield units within an ICH between arterial phase and delayed phase (defined as a 'leakage sign') is even more sensitive for predicting $\mathrm{HE}$ and poor outcome compared to $\mathrm{SS}^{22}$ Another important factor influencing SS sensi- 
tivity for $\mathrm{HE}$ is the contrast phase timing of scanning. The landmark prediction of haematoma growth and outcome in patients with intracerebral haemorrhage using the CT-angiography spot sign (PREDICT) study reported a sensitivity of 51\% of the SS on CTA for HE; however, approximately $20 \%$ of CTAs were actually made in the venous phase. ${ }^{27}$ It has been shown that sensitivity of SS for predicting HE improves when adding a delayed CTA. ${ }^{21}$ In our study we performed bolus-timed injection of contrast media for acquiring the arterial images.

Delayed phase post-contrast $\mathrm{CT}$ acquisition have been shown to not only increase SS detection, but also improve the sensi-

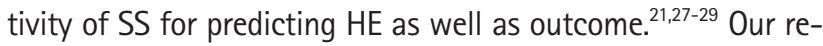
sults are in accordance to these findings with conventionallike mixed delayed phase images more often showing SS as well as having a higher sensitivity for HE when compared to conventional-like mixed arterial phase images. An additional increase in sensitivity, NPV, and accuracy of the SS for HE is seen when DE-CT iodine and fusion datasets are also assessed.

We performed delayed phase imaging at 80 seconds as compromise between persistent venous enhancement and parenchymal enhancement. Other studies reporting on delayed phase, second-pass or multiphase imaging in relation to SS, HE and outcome show a wide distribution in the timing of delayed phase imaging, ranging between 4 and 120 seconds after bolus-timed CTA. ${ }^{21,22,29,30}$ The optimal timing of delayed phase imaging is unknown. There have been concerns of higher radiation dose of DE-CT compared to single-energy $\mathrm{CT}$, but advances in technology have lowered the radiation dose of DE-CT to comparable, or even lower values when compared to singleenergy CT. ${ }^{12,31}$ Therefore, a DE-CT protocol as used in this study, can be safely used in daily clinical practice.

One of the limitations of our study is the high rate of lacking DE-CTA and follow-up CT, mostly due to poor clinical status or demise. This could cause selection bias, and possibly underestimate the amount of hematoma-expanders. This is, however, a reflection of clinical practice and comparable to other studies. ${ }^{21,32}$ Furthermore, SS and HE probably have little extra value in these cases. Lastly, we had irretrievable loss of some of DECTA source data during the course of the study, especially of the delayed fusion images which are missing in 17 patients.

In an attempt to stop or decrease $\mathrm{HE}_{\text {, the }} \mathrm{SS}$ has become a selection tool for therapeutic interventions. Several trials are ongoing, for example the Dutch Intracerebral Hemorrhage Surgery Trial (DIST), Swiss trial of decompressive craniectomy versus best medical treatment of spontaneous supratentorial intracerebral hemorrhage (SWITCH) trial, and Interleukin-1 Receptor Antagonist in Intracerebral Hemorrhage (BLOC-ICH). Applying DE-CTA for the detection of SS in primary ICH pa- tients will gain more and more clinical value and could help to select eligible patients for (future) treatment trials.

\section{Conclusions}

We found that delayed phase fusion imaging has the best diagnostic performance of the SS to predict significant $\mathrm{HE}$, when compared to conventional-like mixed arterial phase imaging, and is associated with poor clinical outcome. Performing DECTA in acute ICH patients, including delayed phase fusion imaging, may help to identify eligible patients for (future) treatment trials (e.g., hemostatic therapy or surgical interventions).

\section{Supplementary materials}

Supplementary materials related to this article can be found online at https://doi.org/10.5853/jos.2020.03531.

\section{Disclosure}

The authors have no financial conflicts of interest.

\section{References}

1. Van Asch CJ, Luitse MJ, Rinkel GJ, van der Tweel I, Algra A, Klijn CJ. Incidence, case fatality, and functional outcome of intracerebral haemorrhage over time, according to age, sex, and ethnic origin: a systematic review and meta-analysis. Lancet Neurol 2010;9:167-176.

2. Xu X, Zhang J, Yang K, Wang O, Xu B, Chen X. Accuracy of spot sign in predicting hematoma expansion and clinical outcome: a meta-analysis. Medicine (Baltimore) 2018;97: e11945.

3. Brouwers HB, Greenberg SM. Hematoma expansion following acute intracerebral hemorrhage. Cerebrovasc Dis 2013;35:195-201.

4. Davis SM, Broderick J, Hennerici M, Brun NC, Diringer MN, Mayer SA, et al. Hematoma growth is a determinant of mortality and poor outcome after intracerebral hemorrhage. Neurology 2006;66:1175-1181.

5. Brott T, Broderick J, Kothari R, Barsan W, Tomsick T, Sauerbeck $L$, et al. Early hemorrhage growth in patients with intracerebral hemorrhage. Stroke 1997;28:1-5.

6. Demchuk AM, Dowlatshahi D, Rodriguez-Luna D, Molina CA, Blas YS, Dzialowski I, et al. Prediction of haematoma growth and outcome in patients with intracerebral haemorrhage using the CT-angiography spot sign (PREDICT): a prospective observational study. Lancet Neurol 2012;11:307-314. 
7. Phan TG, Krishnadas N, Lai VW, Batt M, Slater LA, Chandra $R V$, et al. Meta-analysis of accuracy of the spot sign for predicting hematoma growth and clinical outcomes. Stroke 2019;50:2030-2036.

8. Postma AA, Das M, Stadler AA, Wildberger JE. Dual-energy CT: what the neuroradiologist should know. Curr Radiol Rep 2015;3:16.

9. Watanabe $Y$, Tsukabe $A$, Kunitomi $Y$, Nishizawa $M$, Arisawa $A_{t}$

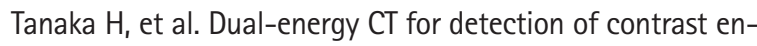
hancement or leakage within high-density haematomas in patients with intracranial haemorrhage. Neuroradiology 2014;56:291-295.

10. Tijssen MP, Hofman PA, Stadler AA, van Zwam W, de Graaf $R$, van Oostenbrugge $\mathrm{RJ}$, et al. The role of dual energy $\mathrm{CT}$ in differentiating between brain haemorrhage and contrast medium after mechanical revascularisation in acute ischaemic stroke. Eur Radiol 2014;24:834-840.

11. Tawfik AM, Kerl JM, Razek AA, Bauer RW, Nour-Eldin NE, VogI TJ, et al. Image quality and radiation dose of dual-energy CT of the head and neck compared with a standard 120kVp acquisition. AJNR Am J Neuroradiol 2011;32:1994-1999.

12. Postma $A A$, Hofman $P A$, Stadler $A A$, van Oostenbrugge RJ, Tijssen MP, Wildberger JE. Dual-energy CT of the brain and intracranial vessels. AJR Am J Roentgenol 2012;199(5 Suppl):S26-S33.

13. Potter CA, Sodickson AD. Dual-energy CT in emergency neuroimaging: added value and novel applications. Radiographics 2016;36:2186-2198.

14. Huynh TJ, Flaherty ML, Gladstone DJ, Broderick JP, Demchuk AM, Dowlatshahi D, et al. Multicenter accuracy and interobserver agreement of spot sign identification in acute intracerebral hemorrhage. Stroke 2014;45:107-112.

15. Kothari RU, Brott $T$, Broderick JP, Barsan WG, Sauerbeck LR, Zuccarello $\mathrm{M}$, et al. The $\mathrm{ABC}$ s of measuring intracerebral hemorrhage volumes. Stroke 1996;27:1304-1305.

16. Tan CO, Lam S, Kuppens D, Bergmans RH, Parameswaran BK, Forghani $R$, et al. Spot and diffuse signs: quantitative markers of intracranial hematoma expansion at dual-energy CT. Radiology 2019;290:179-186.

17. Brouwers HB, Chang Y, Falcone GJ, Cai X, Ayres AM, Battey TW, et al. Predicting hematoma expansion after primary intracerebral hemorrhage. JAMA Neurol 2014;71:158-164.

18. Moon BH, Jang DK, Han YM, Jang KS, Huh R, Park YS. Association factors for $\mathrm{CT}$ angiography spot sign and hematoma growth in Korean patients with acute spontaneous intracerebral hemorrhage: a single-center cohort study. J Korean Neurosurg Soc 2014;56:295-302.

19. Rodriguez-Luna $D$, Rubiera $M$, Ribo $M$, Coscojuela P, Piñeiro
S, Pagola J, et al. Ultraearly hematoma growth predicts poor outcome after acute intracerebral hemorrhage. Neurology $2011 ; 77: 1599-1604$.

20. Kim SH, Jung HH, Whang K, Kim JY, Pyen JS, Oh JW. Which emphasizing factors are most predictive of hematoma expansion in spot sign positive intracerebral hemorrhage? J Korean Neurosurg Soc 2014;56:86-90.

21. Ciura VA, Brouwers HB, Pizzolato R, Ortiz CJ, Rosand J, Goldstein JN, et al. Spot sign on 90-second delayed computed tomography angiography improves sensitivity for hematoma expansion and mortality: prospective study. Stroke 2014;45:3293-3297.

22. Ederies A, Demchuk A, Chia T, Gladstone DJ, Dowlatshahi D, Bendavit $\mathrm{G}$, et al. Postcontrast CT extravasation is associated with hematoma expansion in CTA spot negative patients. Stroke 2009;40:1672-1676.

23. Wada R, Aviv RI, Fox AJ, Sahlas DJ, Gladstone DJ, Tomlinson $\mathrm{G}$, et al. CT angiography "spot sign" predicts hematoma expansion in acute intracerebral hemorrhage. Stroke 2007;38: 1257-1262.

24. Goldstein JN, Fazen LE, Snider R, Schwab K, Greenberg SM,

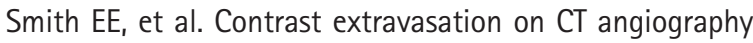
predicts hematoma expansion in intracerebral hemorrhage. Neurology 2007;68:889-894.

25. Han JH, Lee JM, Koh EJ, Choi HY. The spot sign predicts hematoma expansion, outcome, and mortality in patients with primary intracerebral hemorrhage. J Korean Neurosurg Soc 2014;56:303-309.

26. Dowlatshahi D, Brouwers HB, Demchuk AM, Hill MD, Aviv RI, Ufholz LA, et al. Predicting intracerebral hemorrhage growth with the spot sign: the effect of onset-to-scan time. Stroke 2016;47:695-700.

27. Rodriguez-Luna D, Dowlatshahi D, Aviv RI, Molina CA, Silva Y, Dzialowski I, et al. Venous phase of computed tomography angiography increases spot sign detection, but intracerebral hemorrhage expansion is greater in spot signs detected in arterial phase. Stroke 2014;45:734-739.

28. Wu TC, Chen TY, Shiue YL, Chen JH, Hsieh TJ, Ko CC, et al. Added value of delayed computed tomography angiography in primary intracranial hemorrhage and hematoma size for predicting spot sign. Acta Radiol 2018;59:485-490.

29. Rodriguez-Luna D, Coscojuela P, Rodriguez-Villatoro N, Juega JM, Boned S, Muchada M, et al. Multiphase CT angiography improves prediction of intracerebral hemorrhage expansion. Radiology 2017;285:932-940.

30. Kim J, Smith A, Hemphill JC 3rd, Smith WS, Lu Y, Dillon WP, et al. Contrast extravasation on CT predicts mortality in primary intracerebral hemorrhage. AJNR Am J Neuroradiol 
2008;29:520-525.

31. Roele ED, Timmer VC, Vaassen LA, van Kroonenburgh AM, Postma AA. Dual-energy CT in head and neck imaging. Curr Radiol Rep 2017;5:19.

32. Morotti A, Romero JM, Jessel MJ, Brouwers HB, Gupta R,
Schwab K, et al. Effect of CTA tube current on spot sign detection and accuracy for prediction of intracerebral hemorrhage expansion. AJNR Am J Neuroradiol 2016;37:17811786. 
Supplementary Table 1. Baseline characteristics of included primary ICH patients and excluded primary ICH patients

\begin{tabular}{|c|c|c|c|c|c|}
\hline Characteristic & $\begin{array}{l}\text { Data complete } \\
\text { (\% of total) }\end{array}$ & $\begin{array}{l}\text { Total group } \\
(n=235)\end{array}$ & $\begin{array}{l}\text { Included primary ICH patients } \\
\qquad(\mathrm{n}=139)\end{array}$ & $\begin{array}{l}\text { Excluded primary ICH patients* } \\
\qquad(\mathrm{n}=96)\end{array}$ & $P$ \\
\hline \multicolumn{6}{|l|}{ Demographic } \\
\hline Age (yr) & $235(100)$ & $77.0(68.0-83.0)$ & $76.0(65.0-82.0)$ & $78.5(70.3-84.8)$ & 0.024 \\
\hline Male sex & $235(100)$ & $133(56.6)$ & $88(63.3)$ & 45 (46.9) & 0.012 \\
\hline \multicolumn{6}{|l|}{ Clinical } \\
\hline History of hypertension & $233(99.1)$ & $153(65.1)$ & $91(65.5)$ & $62(64.6)$ & 0.938 \\
\hline Hypercholesterolemia & 231 (98.3) & $93(40.3)$ & $54(38.8)$ & $39(40.6)$ & 0.670 \\
\hline Anticoagulant use & $235(100)$ & $62(26.4)$ & $33(23.7)$ & $29(30.2)$ & 0.269 \\
\hline Glucose $(\mathrm{mmol} / \mathrm{L})$ & 226 (96.2) & $7.2(6.0-8.9)$ & $6.7(5.9-8.5)$ & $7.9(6.5-9.7)$ & 0.003 \\
\hline GCS & $235(100)$ & $14(10-15)$ & $15(13-15)$ & $10(5-14)$ & $<0.001$ \\
\hline mRS after 3 months & $220(93.6)$ & $6(3-6)$ & $4(2-6)$ & $6(4-6)$ & $<0.001$ \\
\hline \multicolumn{6}{|l|}{ Imaging } \\
\hline Infratentorial ICH & $235(100)$ & $20(8.5)$ & $9(6.5)$ & $11(11.5)$ & 0.178 \\
\hline Baseline ICH volume (mL) & 234 (99.6) & $16.6(6.5-48.5)$ & $12.0(5.7-27.1)$ & $36.0(8.8-127.1)$ & $<0.001$ \\
\hline Presence of IVH on first CT-scan & $235(100)$ & $103(43.8)$ & 47 (33.8) & 56 (58.3) & $<0.001$ \\
\hline
\end{tabular}

Values are presented as number (\%) or median (interquartile range).

ICH, intracerebral hemorrhage; GCS, Glasgow Coma Scale; mRS, modified Rankin Scale; IVH; intraventricular hemorrhage; CT, computed tomography. *Patients lacking dual-energy computed tomography angiography (DE-CTA) and patients in whom DE-CTA was performed $>3$ hours after baseline CT. 